\title{
The effects of distraction task and initial joint angle time in a joint position matching task
}

\author{
Jungchul Park (jcpark@ut.ac.kr), Sungju Mo (ahtjdwn@naver.com), \\ Hyunmin Yang (hyun9487@gmail.com), Subin Yang (yang94p@naver.com), \\ Suya Jeon (shh500@naver.com)
}

${ }^{1}$ Department of Safety Engineering, Korea National University of Transportation, Chungju, Chungbuk, 27469

\begin{abstract}
Proprioception is a sense that can detect the position and movement of one's own body parts. It is not only essential in the effective interaction with the outside world, but also critical in the situation that bodily movements need to be made in the absence of vision. A joint position matching is a method to evaluate the proprioceptive acuity. In a joint position matching task, subjects should replicate a reference joint angle without the aid of vision, only relying on the proprioceptive information. Then, spatial error between the reference and the matching movement is measured. This study investigates the effect of a distraction task and initial joint angle time on the accuracy of an elbow joint position matching task. An experiment was designed and conducted and twelve participants voluntarily participated in the experiment. In the conditions with distraction tasks, the participants were requested to solve simple calculation problems when they were holding the initial joint angle waiting for the onset of the replication signal. The time for holding the initial joint angle was manipulated as $0 \mathrm{~s}, 5 \mathrm{~s}, 10 \mathrm{~s}$, and $15 \mathrm{~s}$. The results showed that the accuracy of the joint position matching decreased as the initial joint angle time increased. In addition, the effect of distraction task and the interaction between the two factors were significant, showing degraded performance with the presence of the distraction task in most initial joint angle time conditions. A further study is suggested to investigate the effects of different types of distracting tasks in an experiment with a larger scale.
\end{abstract}

Keywords: Proprioception, Joint position matching, Initial joint angle time.

\section{Introduction}

Proprioception means a human sense on one's own body posture, location, balance, and motion. It is received from one's interoceptors in muscles, joints, and tendons. One can recognize the speed and direction of one's own movement by detecting internal stimuli such as contraction and extension of muscles. Proprioception is essential in the physical interaction with the external world (Suetterlin and Sayer, 2014). For example, it may be applied in blind interaction with in-vehicle controls (Choi et al., 2010; Park, 2015) Joint position matching method is used to measure the proprioceptive performance. In this method, a subject is asked to reproduce a reference joint angle without the aid of vision (Goble, 2010).
This paper investigates the effect of a distraction task and initial joint angle time on the accuracy of an elbow joint position matching task.

\section{Method}

An experiment was conducted in order to investigate the effect of a distraction task and initial joint angle time on the accuracy of an elbow joint position matching task. Twelve participants voluntarily participated in the experiment. The participants were seated in a chair and were asked to point a target with their fingertip. The target was located right in front of the participant as high as their shoulder. For each session, they practiced pointing the target for five seconds. After the practice trial, their vision was blocked, and they were requested to hold the initial (relaxed) joint angle for at 
least two seconds. Then, they were asked to perform the pointing task without the aid of vision.at the onset of the replication signal (beep sound). The experimenter marked the location that the participant pointed and measured the distance from the target location.

In the conditions with distraction tasks, the participants were requested to solve simple calculation problems when they were holding the initial joint angle waiting for the onset of the replication signal. The time for holding the initial joint angle was manipulated as $0 \mathrm{~s}, 5 \mathrm{~s}, 10 \mathrm{~s}$, and $15 \mathrm{~s}$. The experiment was within-subjects, that is, every participant performed at all treatment condition. They repeated the same task three times for each condition. The order of presenting the conditions was randomized for every participant.

\section{Results}

Figure 1 shows the mean distance from the target for the eight treatment conditions. The results showed that the accuracy of the joint position matching decreased as the initial joint angle time increased. The effect of the initial joint angle time was significant $(F(3,33)=37.25$, $p<0.0001)$.

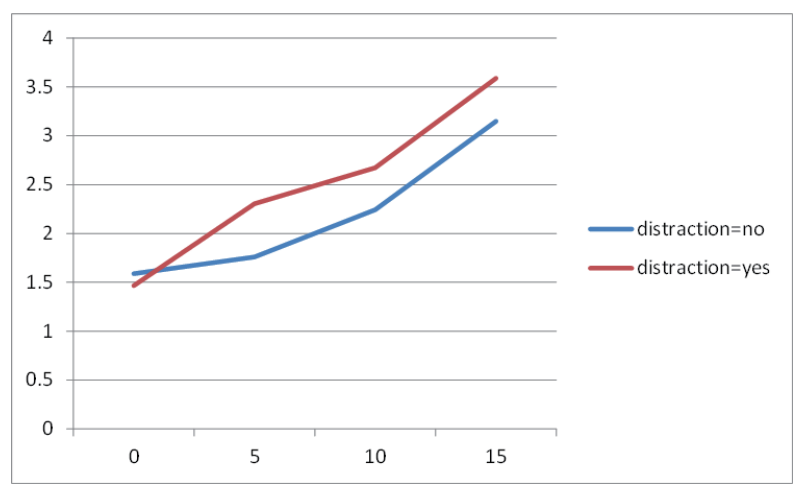

Figure 1. The mean distance from the target for different distraction and initial joint angle time conditions

And, the effect of distraction task was significant, showing degradation of the performance with the presence of distraction task $(F(1,11)=10.46, p=0.008)$. The mean distance was 2.508 for distraction conditions, while it was 2.1841 for non-distraction conditions.

The effect of interaction between the initial joint angle time and the distraction task was also significant
$(F(3,33)=6.67, \quad p=0.0012)$. However, there was no difference in distance between the distraction and non-distraction conditions when the initial joint angle time was 0 second.

\section{Conclusion}

This study investigated the effect of a distraction task and initial joint angle time on the accuracy of an elbow joint position matching task. The results showed that the performance of a joint position matching task is influenced by the duration of initial joint angle time and the presence of distraction task. A further study is suggested to investigate the effects of different types of distracting tasks and different joint angles in an experiment with a larger scale.

\section{Acknowledgements}

This research was supported by Basic Science Research Program through the National Research Foundation of Korea(NRF) funded by the Ministry of Education(NRF-2016R1D1A1B03933470)

\section{References}

Choi, J.Y., Kim, Y.S., Bahn, S.W., Yun, M.H, and Lee M.W. A study on optimal layout of control buttons on center fascia considering human performance under emergency situations. Journal of the Ergonomics Society of Korea, 29(3), 365-373, 2010.

Goble, D.J. Proprioceptive Acuity Assessment via Joint Position Matching: from Basic Science to General Practice, Physical Therapy, 90, 1176-1184, 2010.

Park, J. Factors influencing the proprioceptive acuity of hazard button pressing. In Proceedings of the 2015 Fall Conference of the Ergonomics Society of Korea. 2015.

Suetterlin K.J. and Sayer A.A., Proprioception: where are we now? A commentary on clinical assessment, changes across the life course, functional implications and future interventions, Age and Ageing, 43, 313-318, 2014 